\title{
The Influence of Operational Sex Ratio on the Intensity of Competition for Mates
}

\author{
Laura K. Weir, ${ }^{1, \star}$ James W. A. Grant, ${ }^{2}$ and Jeffrey A. Hutchings ${ }^{3}$ \\ 1. Department of Biological Sciences, Simon Fraser University, Burnaby, British Columbia V5A 1S6, Canada; 2. Department of Biology, \\ Concordia University, Montréal, Québec H4B 1R6, Canada; 3. Department of Biology, Dalhousie University, Halifax, Nova Scotia B3H \\ 4J1, Canada
}

Submitted April 26, 2010; Accepted October 15, 2010; Electronically published January 12, 2011

Online enhancements: appendixes.

\begin{abstract}
AвstRACт: The evolution and maintenance of secondary sexual characteristics and behavior are heavily influenced by the variance in mating success among individuals in a population. The operational sex ratio (OSR) is often used as a predictor of the intensity of competition for mates, as it describes the relative number of males and females who are ready to mate. We investigate changes in aggression, courtship, mate guarding, and sperm release as a function of changes in the OSR using meta-analytic techniques. As the OSR becomes increasingly biased, aggression increases as competitors attempt to defend mates, but this aggression begins to decrease at an OSR of 1.99 , presumably due to the increased costs of competition as rivals become more numerous. Sperm release follows a similar but not significant trend. By contrast, courtship rate decreases as the OSR becomes increasingly biased, whereas mate guarding and copulation duration increase. Overall, predictable behavioral changes occur in response to OSR, although the nature of the change is dependent on the type of mating behavior. These results suggest considerable flexibility of mating system structure within species, which can be predicted by OSR and likely results in variation in the strength of sexual selection.
\end{abstract}

Keywords: mating system, operational sex ratio, contest competition, sperm competition, courtship, mate guarding.

\section{Introduction}

The structure of a mating system can have a profound effect on the mechanisms and strength of sexual selection in a population, and it may influence the evolution and maintenance of secondary sexual characteristics. Mating system structure is typically characterized by the number of mates obtained by the nonlimiting sex of a given species (Selander 1972; Reynolds 1996), and as such it may be considered a fixed characteristic of that species. However,

* Corresponding author; e-mail: lwa45@sfu.ca.

Am. Nat. 2011. Vol. 177, pp. 167-176. (c) 2011 by The University of Chicago. 0003-0147/2011/17702-52115\$15.00. All rights reserved. DOI: $10.1086 / 657918$ considerable flexibility in mating or social systems exists within many species (Lott 1984), and this variability may be attributed to differences in ecological conditions that influence the degree to which mate monopolization can occur (but see Klug et al. 2010). Emlen and Oring (1977) identified the operational sex ratio (OSR; the ratio of potentially receptive males to receptive females at any time; Emlen 1976) as an important ecological factor that can influence mating system structure (e.g., Emlen 1976; Bradbury and Vehrencamp 1977; Thornhill and Alcock 1983). The intensity of both inter- and intrasexual selection is expected to increase as the OSR becomes increasingly biased toward one sex (Emlen and Oring 1977). A biased sex ratio coupled with conventional predictions associated with differential gamete size (Bateman 1948) or parental investment (Trivers 1972), which affect the potential rate of reproduction (PRR; Clutton-Brock and Vincent 1991), often result in intense intrasexual competition, usually among males (Darwin 1871; Huxley 1938; Clutton-Brock and Parker 1992).

Competition for mates can occur through several different mechanisms: scrambles, endurance rivalry, contests, mate choice, and sperm competition (Andersson 1994). Within each of these mechanisms, selection may occur directly, through intrasexual interactions such as fights, or indirectly, through intersexual interactions such as mate choice. Herein, we explore four mechanisms of mate competition that are the most commonly investigated: (1) contests and (2) sperm competition, which occur via direct interactions, and (3) courtship displays and (4) mate guarding or copulation duration, which do not require direct interactions within the nonlimiting sex but which may change due to variation in the relative number of individuals of the two sexes. Our goal is to test current predictions about how different components of mating competition change in response to changes in OSR. Ultimately, this flexibility will affect the variance in the 
strength of sexual selection in different contexts (Shuster 2009) and influence mating system structure.

\section{Direct Competition}

Contest Competition. Contest competition involves fights, chases, and/or threats between competitors, although this varies markedly across species and studies. Aggression usually occurs among males because of differential rates of reproduction between males and females (Trivers 1972; Clutton-Brock and Parker 1992), but it may also occur among females in "sex-role-reversed" species such as Wilson's phalarope (Phalaropus tricolor [Colwell and Oring 1988]). Furthermore, in some species in which the males are usually more aggressive (e.g., sand goby Pomatoschistus minutus [Kvarnemo et al. 1995], Japanese medaka Oryzias latipes [H. Leggett, unpublished observations; Grant et al. 2000; Grant and Foam 2002], European lobster Homarus gammarus [Debuse et al. 1999], two-spotted goby Gobiusculus flavescens [Forsgren et al. 2004], convict cichlid Archocentrus nigrofasciatus [L. Clark, unpublished manuscript]), females can become aggressive if the OSR is female biased. Indeed, intrasexual interactions are likely often occurring among individuals of the limiting sex, albeit at lower levels and in less obvious ways than within the nonlimiting sex (Clutton-Brock 2009).

Emlen and Oring (1977) predicted that a highly biased OSR should result in a decrease in defense, whereas others predict a continuous increase in aggressive or territorial behavior as the OSR becomes increasingly biased (e.g., Tejedo 1988; Kvarnemo and Ahnesjö 1996). While Emlen and Oring (1977) used the OSR as a means of predicting the intensity of competition and mating system structure, they also stressed that the degree of competitive intensity should be considered within the context of "economic defendability" (Brown 1964; Klug et al. 2010). Thus, as resources become less defendable or obtainable-that is, as the OSR becomes heavily biased - animals should invest less energy in contest competition when many competitors are present.

Sperm Competition. Direct competition among sperm can result from a male-biased OSR when there is increased incidence of sneak matings (e.g., Head and Brooks 2006), multiple-male matings (e.g., Byrne and Roberts 2004), and multiple paternity (e.g., Klemme et al. 2007). As such, males might be expected to increase the number of sperm they transfer to the female. Traditionally, sperm had not been considered a major limitation to successful mating (e.g., Bateman 1948; Trivers 1972). Over the past few decades, however, an increasing number of studies have indicated that the production of sperm may be more costly than previously thought (Dewsbury 1982; Nakatsuru and
Kramer 1982; Weir and Grant 2010) and that males may tailor their sperm expenditure according to female status and the size or number of competitors (reviewed in Parker et al. 1997; Wedell et al. 2002). Thus, males may be expected to vary their investment in sperm production and release with respect to the risk (i.e., whether other males are present) as well as the intensity (i.e., how many other males are present; Parker et al. 1996) of sperm competition. Parker et al. (1996) predicted that individual ejaculate expenditure for group spawners would increase as the risk of sperm competition increased (e.g., when two males were present during spawning) and then decrease as the intensity increased.

\section{Indirect Competition}

Courtship. The degree to which animals engage in energetically costly courtship behavior is expected to change as OSR varies. Individuals of many species invest heavily in nuptial coloration and ornamentation (Andersson 1994), but courtship behavior is a more immediately flexible mechanism for mate attraction. At male-biased OSRs, a male's propensity to court females (i.e., proportion of encountered females that are courted; sensu Forsgren et al. 2004) will likely increase. However, the observed frequency of courtship behavior will also be affected by encounter rate with females, which will decrease if the absolute number of females is low (Grant et al. 2000). Regardless of female density, an individual's courtship rate may also decrease as the number of males increases because of increased competition that might result in frequent interruption by other males (Jirotkul 1999) or if males adopt alternative mating strategies other than courtship. However, if courtship is costly in terms of energetic expense or predation risk (e.g., Reynolds 1993), males may invest less energy in courtship as the number of competitors increases.

Mate Guarding and Copulation Duration. The investigation of pre- and postcopulatory mate guarding has focused mainly on invertebrate species, particularly insects and crustaceans. Postcopulatory mate guarding is often linked to paternity assurance, but it may also be involved in mate protection, sperm transfer, or the transfer of nongametic compounds (reviewed by Alcock [1994] for insects). While the duration of guarding varies widely, many studies have documented prolonged contact between a mating pair long after insemination has occurred (Thornhill and Alcock 1983). The duration of postcopulatory mate guarding for paternity assurance is predicted to increase with a greater number of males in a population. It is unlikely that guarding duration will decrease at extremely male-biased OSRs because males who have secured a mate are difficult to 
dislodge and are unlikely to leave in search of another mate if females are rare (Thornhill and Alcock 1983). Copulation duration and/or frequency, which are often considered synonymous with postcopulatory mate guarding, are also expected to increase with increasingly male-biased OSRs. If precopulatory mate guarding is related to males optimizing their time investment, then males should attempt to mate with many different females and invest little time in guarding when females are abundant. However, if females are scarce, males may guard females until they are ready to mate in order to ensure some degree of reproductive success (Parker 1974).

Patterns of Behavior with Changing OSR. For each of the mechanisms discussed above, we tested the following alternative hypotheses regarding patterns of mating behavior as OSR becomes increasingly biased toward one sex: (1) intrasexual aggression will either increase with OSR (Kvarnemo and Ahnesjö 1996; fig. 1A) or peak at intermediate OSRs (Emlen and Oring 1977; fig. 1C); (2) sperm released per mating will either increase with OSR (fig. $1 A$ ) or peak at intermediate OSRs (Parker et al. 1996; fig. 1C); (3) courtship rate will either increase with OSR (fig. 1A), if it is primarily affected by propensity to court (Forsgren et al. 2004), or decrease with OSR (fig. 1B), if it is primarily affected by encounter rate with females (Jirotkul 1999; Grant et al. 2000); and (4) the duration of mate guarding will increase with OSR (Parker 1974; Jormalainen 1998; fig. 1A).

\section{Methods}

\section{Calculating the OSR and Quantifying Behavioral Trends}

Because our data were obtained across a wide range of organisms, we tested for phylogenetic autocorrelation in our data sets using Abouheif's (1999) method. Specifically, we constructed phylogenies for the species within each data set using available data from the Tree of Life Project (http://www.tolweb.org). In addition, we obtained higher resolution for relationships within major clades when necessary (salmonids: Crespi and Fulton 2004; cyprinidontiformes: Hertwig et al. 2008; cypriniformes: Mayden et al. 2009; bitterlings: Fujiwara et al. 2009). We then tested for phylogenetic autocorrelation for discrete traits of either an increase or a decrease in behavior using the Runs Test in Phylogenetic Independence 2.0 (Reeve and Abouheif 2003). This program is designed to test whether a certain trait is significantly associated with its phylogenetic history, and thus it provides guidance as to whether formal comparative analyses are necessary for a given data set. Briefly, this program tests whether an observed $C$ statistic differs significantly from an expected value if the topology of the

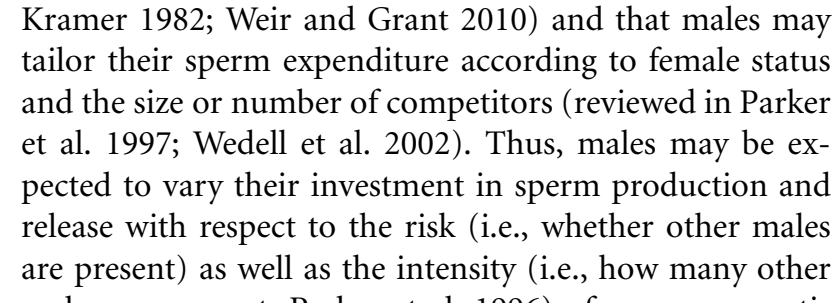

Figure 1: Predicted significant effects of operational sex ratio (OSR) on four components of mating competition. $A$, Continuous increase in the frequency or duration of behavior. $B$, Continuous decrease in the frequency or duration of behavior. $C$, An increase followed by a decrease in the frequency or duration of behavior.

tree were organized at random over a number of iterations. We used 1,000 iterations for each of our four data sets.

Herein, calculations of OSR follow Emlen's (1976) original formulation of the number of mature males to mature females, except in cases of sex-role-reversed species or situations, whereby the OSR is represented as the ratio of mature females to mature males. To standardize the way in which OSR is calculated across studies, some authors (e.g., Kvarnemo and Ahnesjö 1996; Forsgren et al. 2004) represent the OSR as the percentage of males in a population or an experiment. While this method is ideal for experimental studies across a narrow range of OSRs near equality, it can obscure patterns at extremely high or low OSRs. Thus, all estimates of the OSR were recalculated using Emlen's original formulation and plotted on a logarithmic scale. Studies were included if they contained measurements of behavior for at least two values of OSR (app. A in the online edition of the American Naturalist).

To determine overall patterns for each type of mating behavior, we performed formal meta-analyses by calculating effect sizes based on the correlation coefficient $r$ across the maximum and minimum OSR values within each study. Our criteria for including data in the analysis required that the measures of behavior were consistent across studies. Thus, we include studies that report rates or counts of aggressive acts, rates or counts of courtship acts, time of copulation or mate guarding, and the number or rate of sperm released, respectively, for each of our four behavior categories. We also included studies where effect sizes could be calculated from reports of statistical analysis (e.g., $F$ values). Studies that reported data in the form of proportion of time spent in a particular behavior, field studies that were not replicated, and studies that reported the outcome of nonparametric statistics without including means, sample size, or some measure of sample variance were not included in the formal meta-analysis. Our analyses followed methods outlined by Lipsey and Wilson (2001), Harrison et al. (2009), and Jones et al. (2009). 
We standardized effect sizes using Fisher's $Z_{r}$ transform,

$$
E S_{Z_{r}}=0.5 \log _{e}\left(\frac{1+r}{1-r}\right)
$$

where $r$ is the correlation coefficient from a given study. In addition, because we were interested in the direction of change across a range of OSRs, we ensured that effect sizes from each study reflected whether a given behavior was increasing or decreasing as the OSR became increasingly biased toward one sex. As such, we were able to test whether there was an overall increasing or decreasing pattern with OSRs within each study. We assessed the effect of OSRs on mating behavior through metaregression using linear mixed-effects models (LMMs) that were fitted using maximum-likelihood techniques to allow for comparison of fixed effects across different models (Crawley 2007). In all models, we included species as a random factor to account for any effects of pseudoreplication of species across studies. Within each model, we specified withingroup error using the sigma argument with lmeControl, such that error within species with repeated observations was kept constant on the basis of our empirical estimates for each type of behavior. To determine the overall effect of OSR on mating behavior, we assessed whether the intercept from our LMMs differed significantly from 0 using the intercept $t$ value from the model output. We compared models using the Akaike Information Criterion (AIC), beginning with a full model incorporating separate slopes and intercepts within species and removing variables beginning with the slopes for random factors. Effect sizes were weighted to account for differences in sample size (Nakagawa et al. 2007). In addition, we incorporated OSR as a predictor variable to investigate whether the median OSR within a given study affected the direction and strength of effect sizes.

We also investigated whether publication bias may influence the results of our meta-analysis. For each of the four variables investigated, we produced funnel plots for visual inspection of the data. In addition, we determined the rank correlation of effect size and sample size across the studies used in our analysis (Begg and Mazumdar 1994; app. B in the online edition of the American Naturalist). All statistical analyses were performed using S-Plus 6.1 (Insightful). Results were considered to be significant at $\alpha \leq 0.05$.

\section{Results}

We did not find any evidence for phylogenetic autocorrelation for any of the behavior investigated here. For all four data sets, we detected no phylogenetic signal, and the output of Runs Tests indicated that there was a nonsig- nificant tendency for the observed data to show a greater frequency of character change than expected by chance in a random phylogeny, an outcome that is opposite to what would be expected for phylogenetically autocorrelated data (contest competition: $P=.17$; sperm release: $P=.13$; courtship: $P=.13$; mate guarding: $P=.26$ ).

\section{Direct Competition}

Contest Competition. Results of our meta-analysis of 27 studies for 19 species indicated that there was a significant positive effect of increasing OSR on rates of aggression across species $(t=5.61, \mathrm{df}=18, P<.001$; fig. 2$)$. In addition, we detected a significant effect of OSR on the magnitude and direction of effect sizes, such that aggression increased up to a median OSR of 1.99 and then began to decrease $(t=-4.59, \mathrm{df}=7, P=.003$; fig. $3 A)$. Thus, including OSR in our LMM improved the fit of the model (table 1), suggesting that aggression first increases and then decreases as OSR becomes increasingly biased toward one sex.

Sperm Competition. Our meta-analysis of 17 studies suggested that sperm release was not significantly increasing or decreasing overall $(t=0.921, \mathrm{df}=16, P=.37$; fig. 2$)$, nor did OSR influence the direction of the effect size (fig. $3 B$; table 1). Interestingly, six experiments in our data set independently documented an increase-decrease relationship, three of which noted a decrease in sperm investment at an OSR of 2 for an insect, a fish, and a mammal, respectively (northern spring field cricket Gryllus veletis [Schaus and Sakaluk 2001], European bitterling Rhodeus

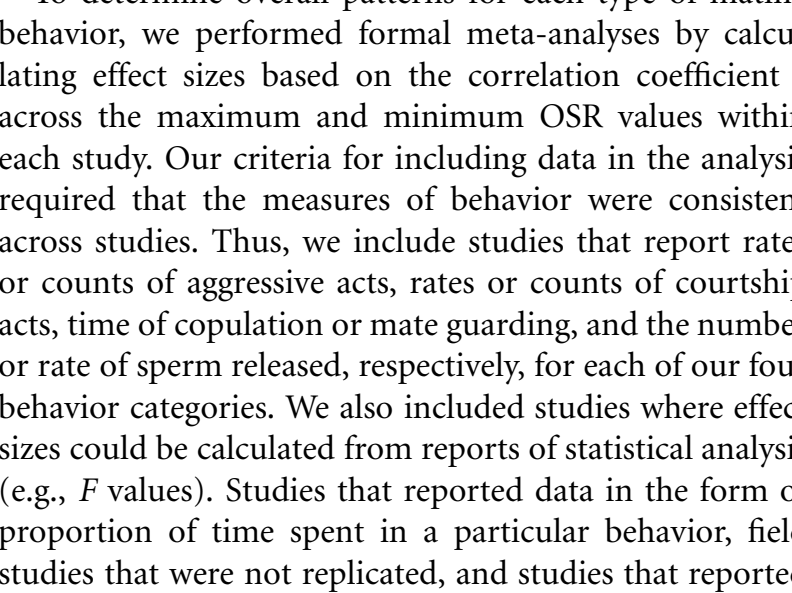

Figure 2: Effect size $(r)$ for direct interactions (filled circles) and indirect competition (open circles) across studies. Error bars represent $95 \%$ confidence limits, and associated values indicate the number of studies used in the analysis. 


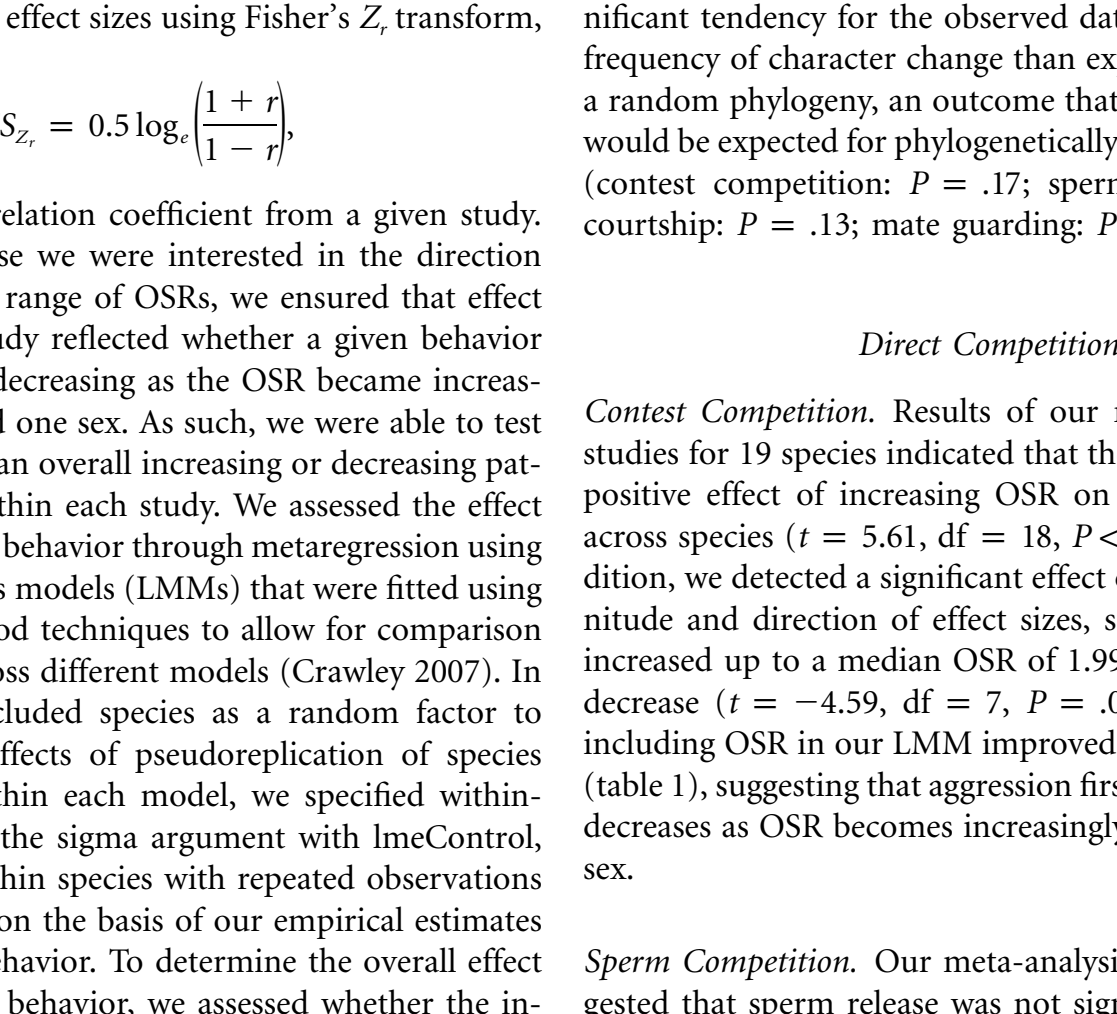

Figure 3: Effect size $(r)$ for each study versus median operational sex ratio (OSR) within study for aggression $(A)$, sperm release $(B)$, courtship rate $(C)$, and mate guarding $(D)$. Error bars in all panels represent $95 \%$ confidence limits within studies. For $A$ and $B$, filled circles denote experiments and open circles denote observational field studies. Regression lines are predicted values from linear models. Inset plots are representative relationships between behavioral frequency and OSR on the basis of the integral of the linear regression line fitted through the data.

sericeus [Candolin and Reynolds 2002a], meadow vole Microtus pennsylvanicus [delBarco-Trillo and Ferkin 2006]), and two experiments showed a decrease in sperm release at a median OSR of 3 (grass goby Zosterisessor ophiocephalus and black goby Gobius niger [Pilastro et al. 2002]). Our calculations obscured increase-decrease relationships, which may affect the overall effect size when these studies are included.

\section{Indirect Competition}

Courtship. The 13 studies included in our meta-analysis indicated a significant overall decrease in courtship rate across studies $(t=-2.68, \mathrm{df}=9, P=.03$; fig. 2$)$. We found no evidence of an effect of OSR on the magnitude or direction of change in courtship (table 1; fig. 3C).

Mate Guarding and Copulation Duration. Overall, the copulation duration effect size across the 19 studies used in our meta-analysis was significantly positive across studies $(t=3.56, \mathrm{df}=18, P=.002$; fig. 2$)$. We found no effect of increasing median OSR in a study on the direction of change in mate guarding and/or copulation duration (table 1; fig. 3D).

\section{Discussion}

\section{Overall Effects of OSR on Mating Behavior}

Our results indicate significant flexibility in mating system structure within species, and they suggest that rigid classification schemes should be interpreted and applied with caution. Three trends emerged from the meta-analysis with respect to changes in behavior with increasing OSR: competitive aggression increased and then decreased, investment in courtship behavior decreased, and mate guarding and copulation duration or attempts increased.

The range in OSR is often broader in observational field studies than in experiments. This trend may be partly due to inaccurate estimation of OSR under natural conditions, whereby the calculated ratio may be more reflective of the adult sex ratio than of the operational sex ratio (Kokko and Jennions 2008). Furthermore, many experiments are 
Table 1: Model selection using Akaike Information Criterion (AIC) criteria for the four mating behaviors in this study

\begin{tabular}{lcccc}
\hline Behavior, predictors & $\mathrm{AIC}_{\mathrm{c}}$ & $K$ & $\Delta \mathrm{AIC}_{\mathrm{c}}$ & $\omega_{\mathrm{AIC}_{\mathrm{c}}}$ \\
\hline Contest competition: & & & & \\
$\quad$ Intercept, OSR & 56.02 & 4 & 0 & $>.99$ \\
$\quad$ Intercept & 74.91 & 3 & 18.89 & $<.01$ \\
Sperm competition: & & & & \\
$\quad$ Intercept, OSR & 56.41 & 4 & 3.37 & .16 \\
$\quad$ Intercept & 53.04 & 3 & 0 & .84 \\
Courtship: & & & & \\
$\quad$ Intercept, OSR & 67.49 & 4 & 9.6 & $<.01$ \\
$\quad$ Intercept & 57.93 & 3 & 0 & $>.99$ \\
Mate guarding and & & & & \\
$\quad$ copulation duration: & & & & \\
$\quad$ Intercept, OSR & 145.19 & 3 & 100.49 & $<.01$ \\
$\quad$ Intercept & 44.69 & 2 & 0 & $>.99$ \\
\hline
\end{tabular}

Note: $\mathrm{AIC}_{\mathrm{c}}=$ corrected AIC values to account for small sample size, $K=$ number of parameters, $\Delta \mathrm{AIC}_{c}=$ the difference in $\mathrm{AIC}_{c}$ between models, and $\omega_{\mathrm{AIC}_{\mathrm{c}}}=$ Akaike weight of each model.

designed to test female-biased, equal, and male-biased sex ratios, which are usually $1: 2,1: 1$, and $2: 1$, respectively, such that extremely biased OSRs are often not investigated. It is likely that the narrower range of OSRs in experimental studies is partly a result of logistic difficulties associated with studying extremely biased OSRs, which require higher numbers of competitors and larger-scale experimental arenas. In addition, studies of sperm competition, as well as of mate guarding and copulation duration, are very difficult to undertake at larger scales. Indeed, competition occurs locally, and as such, small-scale experiments may lend more insight into what happens during mating in the wild than one might assume under "unrealistic" experimental conditions (see Gwynne et al. 1998). Regardless, combining experimental and field data has identified important trends in mating behavior with respect to OSR.

\section{Direct Competition}

Interestingly, contest competition followed an increasedecrease pattern with respect to increasing OSR. This result is consistent with predictions made by Emlen and Oring (1977), and it suggests a switch from an increase to a decrease in competitive intensity at an OSR of 2. While highly biased OSRs are defined by an increase in competitive intensity, the form of competition may change from interference competition, characterized by aggression, to scramble competition, characterized by the searching for and securing of mates (Grant et al. 2000; Klug et al. 2010). While we did not detect an effect of OSR on sperm competition, Parker et al. (1996) predicted that sperm release should increase and then decrease with an increasing number of competitors in externally fertilizing species. Three studies used in our analysis support this prediction, but we did not find an overall effect of OSR on sperm release, nor did we detect a consistent increase or decrease in sperm release overall. While visual inspection of our data suggests an increase-decrease trend (fig. 3 ), it is likely that sample size was too small and withinstudy variance was too large to detect an effect. Because of our conservative analysis, increase-decrease relationships may be obscured. Furthermore, dominant and subordinate males may respond differently to increases in OSR. For example, a highly male-biased OSR may result in an increase in aggression by subordinates due to a relaxation of aggressive interactions initiated by dominant individuals (Mills and Reynolds 2003). Similarly, an increase in sperm release frequency for smaller, subordinate European bitterling males at highly male-biased OSRs is coupled with a decrease in sperm release frequency for larger, territorial males (Mills and Reynolds 2003). These empirical results support recent work by Klug et al. (2010), whereby a heavily biased OSR is likely to decrease the degree of monopolizability of mates and decrease variance in reproductive success in a population.

\section{Indirect Competition}

Courtship rate decreased and mate guarding increased as OSR became increasingly biased. Because of the nature of these interactions, these trends may be driven by the relative availability of the limiting sex rather than the number of same-sex competitors. Grant et al. (2000) suggest that the overall decrease in courtship behavior by males at a highly male-biased OSR is most likely the result of a lack of availability of females to court. In some species, specific types of courtship behavior are energetically demanding, such that there may be a switch in the type of courtship behavior that males exhibit (Shine et al. 2003). At high male density, some authors identify a trade-off between courtship and other mating behavior, such as sneaky matings (Kanoh 2000; Mills and Reynolds 2003).

The duration of mate guarding by males increased with increasingly male-biased OSRs. However, it is important to distinguish the mechanisms resulting in pre- and/or postcopulatory mate guarding or copulation duration. Most studies that examine precopulatory mate guarding involve species for which the duration of female receptivity to mate is brief (Iribarne et al. 1995; Jormalainen and Shuster 1999; Wada et al. 1999; Rondeau and Sainte-Marie 2001; Mathews 2002; Kamio et al. 2003). In these cases, males mate guard to (1) wait for the female to become receptive; (2) ensure paternity, either by preventing females from remating before oviposition or by transferring as many sperm as possible to the female; or (3) obtain future copulation opportunities. Thus, if females are rare, 
it is expected that males should remain with a mate and forgo the search for a new one. However, if females are abundant and encounters are frequent, males may abandon females who are not receptive and attempt to find one who is ready to mate (Parker 1974). Furthermore, mate guarding following copulation may prevent sperm competition from subsequent males.

\section{Relative Effects of OSR and Density}

Changes in the intensity of competition may occur because animals are assessing (1) the absolute number of competitors, (2) the absolute number of mates, or (3) the relative number of competitors with respect to the abundance of a resource. Because OSR is a ratio variable, it is influenced by both the number of same-sex competitors and the number of potential mates. These two components may have different effects on behavior depending on how individuals assess mating opportunities. The exact cause of a change in OSR under natural conditions is likely context and species dependent. For example, Moore (1987) suggested that changes in the OSR of dragonflies occurred because of a change in male density and not the number of females on a pond.

Manipulation of OSR usually involves a change in overall density, because most experimental treatments vary the number of individuals of the competing sex while holding the limiting sex at a constant number (e.g., Joy and Crews 1985; Candolin and Reynolds 2002a, 2002b; Pilastro et al. 2002; but see Kodric-Brown 1988; Souroukis and Cade 1993 for the converse). By increasing the number of individuals of the competing sex, there may be a density effect that is independent of OSR due to an increase in an encounter rate that may result in more interactions. However, in most cases investigated here, the change caused by the manipulation of both factors was additive. In some studies, males responded to both changes in competitor density and mate number (Arnqvist 1992; Iribarne et al. 1995; Alonso-Pimentel and Papaj 1996), while in others, the number of mates was the most important factor (Kvarnemo et al. 1995; Smith 2007). The only significant interaction between sex ratio and total density on male aggression and male courtship rates was documented in experiments by Spence and Smith (2005), where changes in male aggression occurred at only low density and changes in male courtship rates occurred at high density. In general, however, when the effect of density was considered in experiments, density affected the magnitude of the effect of OSR but not the directional change in competitive behavior (e.g., Arnqvist 1992; Alonso-Pimentel and Papaj 1996). Even when density was identified as being more important than OSR per se, the directional changes in behavior have typically been consistent with predictions of OSR theory (e.g., Verrell 1982; Sih and Krupa 1995). As such, our analyses have likely captured the true effects of OSR on behavior rather than a density effect due to an increased probability of interactions.

\section{Conclusions}

Our results suggest that the intensity of mating competition does not always increase with increasing OSR. We found significant flexibility in the mating behavior examined, which suggests considerable flexibility in mating system structure for many species. In direct competition, patterns of contest competition indicate that individuals react to OSR in an energetically economical way. This overlying trend is different from what some authors have predicted (e.g., Kvarnemo and Ahnesjö 1996), but it is consistent with the predictions of Emlen and Oring (1977) on the basis of resource-defense theory. While we did not detect an effect of OSR on sperm release, the trend in the data was very similar to that for for contest competition, and some authors suggest that males may be conservative in their release of sperm when OSR becomes increasingly male biased, such that individuals release fewer sperm as the number of competitors increases (as predicted in Parker et al. 1996). Thus, when competing for access to mates through very direct means, animals appear to act to conserve energy and resources under conditions that would not allow for economic defense of females.

By contrast, behavior involved in indirect competition changed in a consistent direction as OSR increased. Courtship rate tended to decrease as OSR increased, whereas mate guarding duration increased with increasing OSR as males attempted to secure mates rather than search for new ones. As discussed above, courtship is likely a costly behavior that is readily decreased when the level of competition is too high, in part because females may not be available to receive courtship and competing males interrupt male courtship behavior. Lack of availability of receptive females at male-biased OSRs is likely driving the increase in mate guarding or copulation duration, such that males maintain contact with females once they are located.

We find that OSRs can have a direct influence on the intensity of competition for mates, which is typically linked to the strength of intrasexual selection. However, other determinants of mating systems, such as parental care and sex roles, may be influenced by factors other than the OSR (Kokko and Monaghan 2001; Kokko and Jennions 2008). Furthermore, it is important to distinguish the OSR from the adult sex ratio (ASR) and the relative influence these factors may have over the evolution of different components of mating systems. Kokko and Jennions (2008), for example, found that ASR may be a better predictor of 
parental investment than OSR. As they discussed, experimental studies often equate OSR and ASR, while the relationship between these two variables is likely to vary under natural conditions.

Although the results of this study indicate that OSR can influence mating behavior, other ecological and environmental factors may affect both the adult sex ratio and the OSR, and they can also affect mating behavior independent of changes in OSR. For example, one sex may be more susceptible to predation, such that under different predation regimes, adult sex ratios in the same species may be more biased (e.g., McKellar et al. 2009). Furthermore predation may influence the type of behavior that some individuals will use to gain access to mates. For example, if predation is intense, males may forgo conspicuous court. ship displays and attempt to gain some reproductive success by sneak matings. In addition, resource availability, especially when considering resources required for reproduction, may affect the number of individuals in a population that are available for reproduction (e.g., Lindström 2001; Debuse et al. 2003). Thus, ecological or environmental factors may influence how OSR changes, as wel $\rightarrow$ as how behavior may change across a range of OSRs in a given population or species.

Overall, variability in mating behavior in different contexts suggests that mating system structure may be affected by changes in external factors such as the OSR, which is often used as a proxy for the intensity of sexual selection Thus, the OSR may ultimately influence the distribution and variance in reproductive success among individual in a population, and it may be a strong driving force in the evolution and maintenance of sexual characteristics.

\section{Acknowledgments}

We are grateful to P. Blanchfield and H. Leggett for providing unpublished data. We thank B. Crespi, M. Johnston, B. Jonsson, M. Leonard, H. Whitehead, and anonymous referees for comments on earlier versions of this manuscript. W. Blanchard, A. Cooper, and an anonymous referee provided advice for statistical analyses. This work was funded by National Sciences and Engineering Research Council (NSERC) grants to J.W.A.G. and J.A.H. ani $\rightarrow$ NSERC Fonds Québécois de la Recherche sur la Nature et les Technologies and Killam Trust scholarships to L.K.W.

\section{Literature Cited}

Abouheif, E. 1999. A method for testing the assumption of phylogenetic independence in comparative data. Evolutionary Ecology Research 1:895-909.

$\rightarrow$ Alcock, J. 1994. Postinsemination associations between males and females in insects: the mate-guarding hypothesis. Annual Review of Entomology 39:1-21.

Alonso-Pimentel, H., and D. R. Papaj. 1996. Operational sex ratio versus gender density as determinants of copulation duration in the walnut fly, Rhagoletis juglandis (Diptera: Tephritidae). Behavioral Ecology and Sociobiology 39:171-180.

Andersson, M. 1994. Sexual selection. In J. R. Krebs and T. CluttonBrock, eds. Monographs in behavior and ecology. Princeton University Press, Princeton, NJ.

Arnqvist, G. 1992. Pre-copulatory fighting in a water strider: intersexual conflict or mate assessment? Animal Behaviour 43:559-567.

Bateman, A. J. 1948. Intra-sexual selection in Drosophila. Heredity 2:349-368.

Begg, C. R., and M. Mazumdar. 1994. Operating characteristics of a rank correlation test for publication bias. Biometrics 50:10881101.

Bradbury, J. W., and S. L. Vehrencamp. 1977. Social organization and foraging in emballonurid bats. III. Mating systems. Behavioral Ecology and Sociobiology 2:1-17.

Brown, J. L. 1964. The evolution of diversity in avian territorial systems. Wilson Bulletin 76:160-169.

Byrne, P. G., and J. D. Roberts. 2004. Intrasexual selection and group spawning in quacking frogs (Crinia georgiana). Behavioral Ecology 15:872-882.

Candolin, U., and J. D. Reynolds. 2002a. Adjustments of ejaculation rates in response to risk of sperm competition in a fish, the bitterling (Rhodeus sericeus). Proceedings of the Royal Society B: Biological Sciences 269:1549-1553.

. 2002b. Why do males tolerate sneakers? tests with the European bitterling, Rhodeus sericeus. Behavioral Ecology and Sociobiology 51:146-152.

$\rightarrow$ Clutton-Brock, T. H. 2009. Sexual selection in females. Animal Behaviour 77:3-11.

$\rightarrow$ Clutton-Brock, T. H., and G. A. Parker. 1992. Potential reproductive rates and the operation of sexual selection. Quarterly Review of Biology 67:437-456.

$\rightarrow$ Clutton-Brock, T. H., and A. J. Vincent. 1991. Sexual selection and the potential reproductive rates of males and females. Nature 351 : 58-60.

$\rightarrow$ Colwell, M. A., and L. W. Oring. 1988. Sex ratios and intrasexual competition in a sex-role reversed shorebird, Wilson's phalarope (Phalaropus tricolor). Behavioral Ecology and Sociobiology 22:165173.

Crawley, M. J. 2007. The R book. Wiley, Chichester.

$\rightarrow$ Crespi, B. J., and M. J. Fulton. 2004. Molecular systematic of Salmonidae: combined nuclear data yields a robust phylogeny. Molecular Phylogenetics and Evolution 31:658-679.

Darwin, C. 1871. The descent of man and selection in relation to sex. Princeton University Press, Princeton, NJ.

Debuse, V. J., J. T. Addison, and J. D. Reynolds. 1999. The effects of sex ratio on sexual competition in the European lobster. Animal Behaviour 58:473-481.

$\rightarrow \longrightarrow$. 2003. Effects of breeding site density on competition and sexual selection in the European lobster. Behavioral Ecology 14: 396-402.

$\rightarrow$ delBarco-Trillo, J., and M. H. Ferkin. 2006. Male meadow voles respond differently to risk and intensity of sperm competition. Behavioral Ecology 17:581-585.

$\rightarrow$ Dewsbury, D. A. 1982. Ejaculate cost and male choice. American Naturalist 119:601-610. 
$\rightarrow$ Emlen, S. T. 1976. Lek organization and mating strategies in the bullfrog. Behavioral Ecology and Sociobiology 283:283-313.

$\rightarrow$ Emlen, S. T., and L. W. Oring. 1977. Ecology, sexual selection anc $\rightarrow$ K the evolution of mating systems. Science 197:215-223.

$\rightarrow$ Forsgren, E., T. Amundsen, A. A. Borg, and J. Bjelvenmark. 2004. Unusually dynamic sex roles in a fish. Nature 429:551-554.

$\rightarrow$ Fugiwara, M., J. Inafuku, A. Takeda, A. Watanabe, A. Fujiwara, S. Kohno, and S. Kubota. 2009. Molecular organization of 5S rDNA in bitterlings (Cyprinidae). Genetica 135:355-365.

$\rightarrow$ Grant, J. W. A., and P. E. Foam. 2002. Effect of operational sex ratio on female-female versus male-male competitive aggression. Canadian Journal of Zoology 80:2242-2246.

$\rightarrow$ Grant, J. W. A., C. L. Gaboury, and H. L. Levitt. 2000. Competitorto-resource ratio, a general formulation of operational sex ratio, $\rightarrow$ as a predictor of competitive aggression in Japanese medaka (Pisces: Oryziidae). Behavioral Ecology 11:670-675.

$\rightarrow$ Gwynne, D. T., J. T. Bailey, and A. Annells. 1998. The sex in shor $\rightarrow$ supply varies over small spatial scales in a katydid (Kawanaphila nartee, Orthoptera: Tettigoniidae). Behavioral Ecology and Sociobiology 42:157-162.

$\rightarrow$ Harrison, F., Z. Barta, I. Cuthill, and T. Székeley. 2009. How is sexual conflict over parental care resolved? a meta-analysis. Journal of Evolutionary Biology 22:1800-1812.

$\rightarrow$ Head, M. L., and R. Brooks. 2006. Sexual coercion and the opportunity for sexual selection in guppies. Animal Behaviour 71:515- $\rightarrow$ 522.

$\rightarrow$ Hertwig, S. T. 2008. Phylogeny of the Cyprinodontiformes (Teleostei $\rightarrow$ Atherinomorpha): the contribution of cranial soft tissue characters. Zoologica Scripta 37:141-174.

$\rightarrow$ Huxley, J. S. 1938. Darwin's theory of sexual selection and the data subsumed by it, in the light of recent research. American Naturalist 72:416-473.

$\rightarrow$ Iribarne, O., M. Fernandez, and D. Armstrong. 1995. Precopulatory guarding-time of the male amphipod Eogammarus oclairi: effect of population structure. Marine Biology 124:219-223.

$\rightarrow$ Jirotkul, M. 1999. Operational sex ratio influences female preferenci and male-male competition in guppies. Animal Behaviour 58:287294.

$\rightarrow$ Jones, K. S., S. Nakagawa, and B. Sheldon. 2009. Environmental sensitivity in relation to size and sex in birds: meta-regression analysis. American Naturalist 174:122-133.

$\rightarrow$ Jormalainen, V. 1998. Precopulatory mate guarding in crustaceans $\rightarrow$ Mo male competitive strategy and intersexual conflict. Quarterly Review of Biology 73:275-304.

$\rightarrow$ Jormalainen, V., and M. Shuster. 1999. Female reproductive cycle and sexual conflict over precopulatory mate-guarding in Ther $\rightarrow$ mosphaeroma (Crustacea, Isopoda). Ethology 105:233-246.

$\rightarrow$ Joy, J. E., and D. Crews. 1985. Social dynamics of group courtship behaviour in male red-sided garter snakes (Thamnophis sirtalis parietalis). Journal of Comparative Psychology 99:145-149.

$\rightarrow$ Kamio, M., S. Matsunaga, and N. Fusetani. 2003. Observation on the mating behaviour of the helmet crab Telmessus cheiragonus (Brachyura: Cheiragonidae). Journal of the Marine Biological As $\rightarrow$ sociation of the United Kingdom 83:1007-1013.

$\rightarrow$ Kanoh, Y. 2000. Reproductive success associated with territoriality, $\rightarrow$ sneaking, and grouping in male rose bitterlings, Rhodeus ocellatus (Pisces: Cyprinidae). Environmental Biology of Fishes 57:143-154.

$\rightarrow$ Klemme, I., H. Ylönen, and J. A. Eccard. 2007. Reproductive success of male bank voles (Clethrionomys glareolus): the effect of oper $\rightarrow$ ational sex ratio and body size. Behavioral Ecology and Sociobiology 61:1911-1918.

Klug, H., J. Heuschele, M. D. Jennions, and H. Kokko. 2010. The mismeasurement of sexual selection. Journal of Evolutionary Biology 23:447-462.

$\rightarrow$ Kodric-Brown, A. 1988. Effects of sex-ratio on territoriality and spawning success of male pipefish, Cyprinodon pecosensis. Animal Behaviour 36:1136-1144.

$\rightarrow$ Kokko, H., and M. D. Jennions. 2008. Parental investment, sexual selection and sex ratios. Journal of Evolutionary Biology 21:919948.

$\rightarrow$ Kokko, H., and P. Monaghan. 2001. Predicting the direction of sexual selection. Ecology Letters 4:159-165.

$\rightarrow$ Kvarnemo, C., and I. Ahnesjö. 1996. The dynamics of operational sex ratios and competition for mates. Trends in Ecology \& Evolution 11:404-408.

Kvarnemo, C., E. Forsgren, and C. Magnhagen. 1995. Effects of sex ratio on intra- and inter-sexual behaviour in sand gobies. Animal Behaviour 50:1455-1461.

$\rightarrow$ Lindström, K. 2001. Effects of resource distribution on sexual selection and the cost of reproduction in sandgobies. American Naturalist 158:64-74.

Lipsey, M. W., and D. B. Wilson. 2001. Practical meta-analysis. Applied Social Research Methods Series. SAGE, Thousand Oaks, CA.

Lott, D. L. 1984. Intraspecific variation in the social systems of wild vertebrates. Behaviour 88:266-325.

Mathews, L. M. 2002. Tests of the mate-guarding hypothesis for social monogamy: does population density, sex ratio, or female synchrony affect behaviour of male snapping shrimp (Alpheus angulatus)? Behavioral Ecology and Sociobiology 51:426-432.

Mayden, R. L., K. L. Tang, R. M. Wood, W.-J. Chen, M. K. Agnew, K. W. Conway, L. Yang, et al. 2008. Inferring the tree of life of the order Cypriniformes, the earth's most diverse clade of freshwater fishes: implications of varied taxon and character sampling. Journal of Systematics and Evolution 46:424-438.

McKellar, A. E., M. M. Turcotte, and A. P. Hendry. 2009. Environmental factors influencing adult sex ratio in Trinidadian guppies. Oecologia (Berlin) 159:735-745.

Mills, S. C., and J. D. Reynolds. 2003. Operational sex ratio and alternative reproductive behaviours in the European bitterling, Rhodeus sericeus. Behavioral Ecology and Sociobiology 54:98-104.

Moore, A. J. 1987. The behavioural ecology of Libellula luctuosa (Burmeister) (Anisoptera: Libellulidae). I. Temporal changes in the population density and the effects on male territorial behavior. Ethology 75:246-254.

$\rightarrow$ Nakagawa, S., N. Ockendon, G. O. S. Gillespie, B. J. Hatchwell, and T. Burke. 2007. Assessing the function of house sparrow's bib size using a flexible meta-analysis method. Behavioral Ecology 18:831840.

$\rightarrow$ Nakatsuru, K., and D. L. Kramer. 1982. Is sperm cheap? limited male fertility and female choice in the lemon tetra (Pisces, Characidae). Science 216:753-755.

Parker, G. A. 1974. Courtship persistence and female-guarding as male time investment strategies. Behaviour 48:157-184.

Parker, G. A., M. A. Ball, P. Stockley, and M. J. G. Gage. 1996. Sperm competition games: individual assessment of sperm competition intensity by group spawners. Proceedings of the Royal Society B: Biological Sciences 263:1291-1297.

$\rightarrow+1997$. Sperm competition games: a prospective analysis of 
risk assessment. Proceedings of the Royal Society B: Biological Sciences 264:1793-1802.

$\rightarrow$ Pilastro, A., M. Scaggiante, and M. B. Rasotto. 2002. Individual adjustment of sperm expenditure accords with sperm competitior theory. Proceedings of the National Academy of Sciences of the USA 99:9913-9915.

Reeve, J., and E. Abouheif. 2003. Phylogenetic independence. Ver $\rightarrow$ Spen 2.0. http://biology.mcgill.ca/faculty/abouheif/programs_pi.htm.

$\rightarrow$ Reynolds, J. D. 1993. Should attractive individuals court more? theory and a test. American Naturalist 141:914-927.

$\rightarrow$. 1996. Animal breeding systems. Trends in Ecology \& Evolution 11:68-72.

$\rightarrow$ Rondeau, A., and B. Sainte-Marie. 2001. Variable mate-guarding time and sperm allocation by male snow crabs (Chionecetes opilio) in response to sexual competition, and their impact on the mating success of females. Biological Bulletin 201:204-217.

$\rightarrow$ Schaus, J. M., and S. K. Sakaluk. 2001. Ejaculate expenditures of male crickets in response to varying risk and intensity of sperm com petition: not all species play games. Behavioral Ecology 12:740745.

Selander, R. K. 1972. Sexual selection and dimorphism in birds. Page 180-230 in B. Campbell, ed. Sexual selection and the descent of man, 1871-1971. Aldine, Chicago.

$\rightarrow$ Shine, R., T. Langkilde, and R. T. Mason. 2003. The opportunistic serpent: male garter snakes adjust courtship tactics to mating opportunities. Behaviour 140:1509-1526.

Shuster, S. M. 2009. Sexual selection and mating systems. Proceedings of the National Academy of Sciences of the USA 106(suppl.): 10009-10016.

$\rightarrow$ Sih, A., and J. J. Krupa. 1995. Interacting effects of predation risk and male and female density on male/female conflicts and mating dynamics of stream water striders. Behavioral Ecology 6:316-325.

$\rightarrow$ Smith, C. C. 2007. Independent effect of male and female density on sexual harassment, female fitness, and male competition for mates in the western mosquitofish Gambusia affinis. Behavioral Ecology and Sociobiology 61:1349-1358.

Souroukis, K., and W. H. Cade. 1993. Reproductive competition and selection on male traits at varying sex ratios in the field cricket, Gryllus pennsylvanicus. Behaviour 126:45-62.

Spence, R., and C. Smith. 2005. Male territoriality mediates density and sex ratio effects on oviposition in the zebrafish, Danio rerio. Animal Behaviour 69:1317-1323.

$\rightarrow$ Tejedo, M. 1988. Fighting for females in the toad Bufo calamita is affected by the operational sex ratio. Animal Behaviour 36:17651769.

Thornhill, R., and J. Alcock. 1983. The evolution of insect mating systems. Harvard University Press, Cambridge, MA.

Trivers, R. L. 1972. Parental investment and sexual selection. Pages 136-179 in B. Campbell, ed. Sexual selection and the descent of man, 1871-1971. Aldine, Chicago.

$\rightarrow$ Verrell, P. 1982. The sexual behavior of the red spotted newt, Notophthalmus viridescens (Amphibia, Urodela, Salamandridae). Animal Behaviour 30:1224-1236.

Wada, S., K. Tanaka, and S. Goshima. 1999. Precopulatory mate guarding in the hermit crab, Pagurus middendorffii (Brandt) (Decopoda: Paguridae): effects of population parameters on male guarding duration. Journal of Experimental Marine Biology and Ecology 239:289-298.

$\rightarrow$ Wedell, N., M. J. G. Gage, and G. A. Parker. 2002. Sperm competition, male prudence and sperm-limited females. Trends in Ecology \& Evolution 17:313-320.

$\rightarrow$ Weir, L. K., and J. W. A. Grant. 2010. Courtship signals fertility in an externally fertilizing fish. Biology Letters 6:727-731.

Associate Editor: Suzanne H. Alonzo Editor: Ruth G. Shaw 